Le « code de conduite " politique du Nandi Kaburwo Council of Elders : libérer la communauté nandi d'un mauvais sort en réhabilitant les « bons leaders » en temps électoral

Chloé Josse-Durand

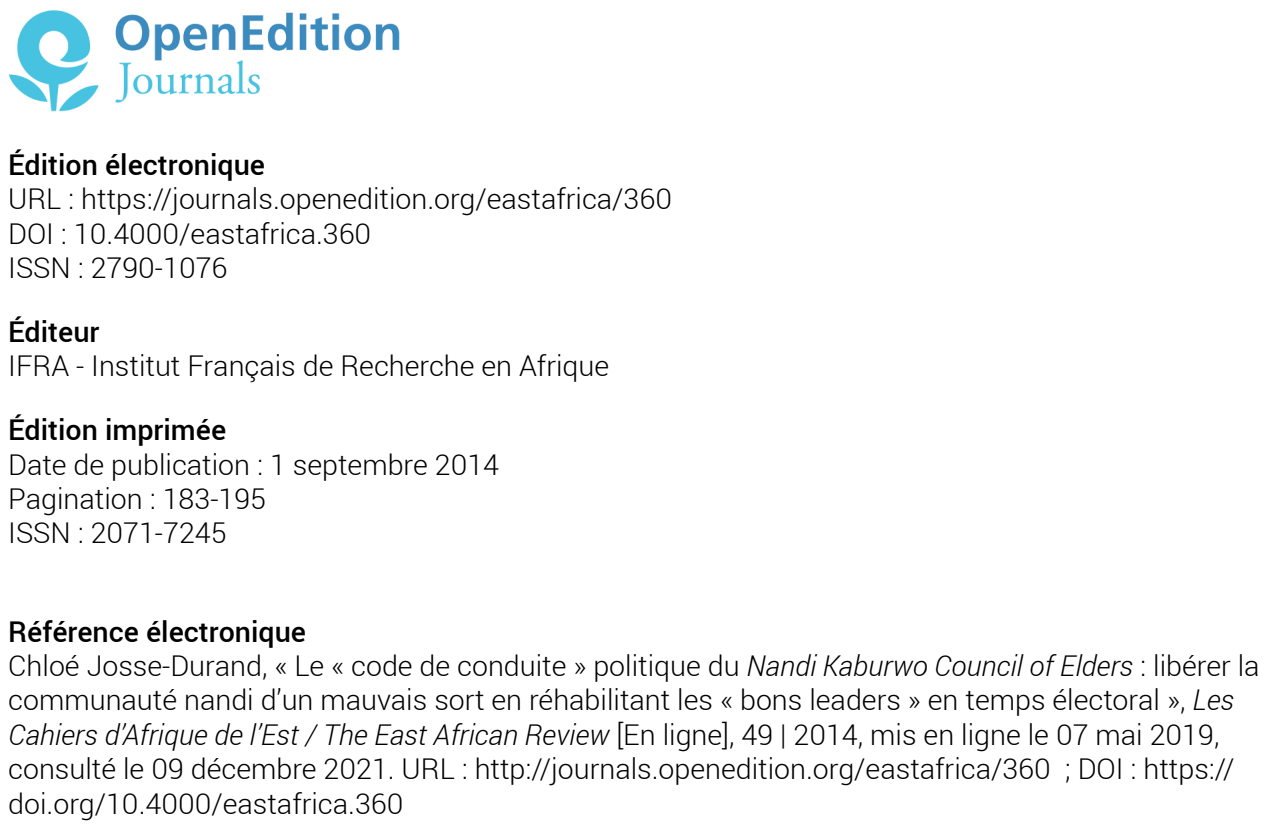




\title{
Le « code de conduite » politique du Nandi Kaburwo Council of Elders : libérer la communauté nandi d'un mauvais sort en réhabilitant les « bon leaders » en temps électoral
}

\author{
Chloé Josse-Durand
}

Alors que je menais une mission de recherche ${ }^{3}$ dans le comté Nandi en mars 2013, la campagne politique pour les élections 2013 battait son plein : les meetings s'enchaînaient sur le petit square central de la ville de Nandi Hills et, non loin de là, les marchands de journaux avaient installé des bancs, invitant les lecteurs à prendre le temps d'ouvrir le Daily Nation, le Standard ou encore le Kass, le magazine régional en langue kalenjin. Quelques militants, employés par les partis politiques pour s'affronter en joutes verbales dans différents endroits passants de la ville, s'étaient accaparé certains de ces bancs et les avaient réorganisés en carré où, arborant t-shirts et casquettes aux couleurs de leur parti, ils affrontaient verbalement les agents du parti adverse. À quelques jours de l'élection de six représentants d'importance (le président, le député, le sénateur, le gouverneur de comté, la représentante des femmes et le conseiller local), tensions et espoirs alimentaient les compétitions politiques alors que le nouveau découpage administratif donnait un rôle plus important aux collectivités territoriales. Cette élection pouvait être l'occasion d'un renouvellement de la classe politique en termes partisans mais également en termes générationnels.

Pourtant, loin de cette effervescence, d'autres acteurs avaient abordé la campagne de façon plus rationnelle, sur la base de stratégies élaborées en amont avec des objectifs bien précis. Le Nandi Kaburwo Council of Elders, ${ }^{4}$ une association qui se présente comme un regroupement des anciens et des sages de la communauté nandi, avait en effet entrepris la rédaction d'un code de conduite politique plus d'un an avant la tenue des

3 Cette mission de trois semaines, réalisée avec le soutien financier de l'IFRA-Nairobi, a été effectuée dans le cadre de ma thèse entamée en 2011 sur les enjeux politiques et identitaires qui accompagnent la création d'un musée-mausolée dédié à un héros local, Koitalel Samoei, à Nandi Hills, Nandi County.

4 Kaburwo m'a été traduit par les elders par « the shade of the tree », qui a pour vocation de rappeler les palabres que les anciens tenaient à l'ombre des ficus centenaires, anciens lieux sociaux et religieux respectés par les nandis. Ce terme explique le symbole choisi pour représenter le Nandi Kaburwo Council of Elders, un arbre aux larges branches que l'on retrouve sur la couverture du NCPCC. 
élections, le Nandi County Political Code of Conduct ${ }^{5}$ (NCPCC). Destiné aux candidats du comté, à leurs partisans et à leur parti, ce code de conduite énonce un certain nombre de règles censées contribuer à transformer le comté Nandi en un îlot de prospérité, " the island of new politics, free of political madness $\gg .{ }^{6}$ Adopté en avril 2012, le NCPCC a été diffusé par le Nandi Kaburwo Council of Elders avec le soutien du Center for Community Dialogue and Development (CCDD), une ONG promouvant les elders comme vecteurs d'unité au sein de leur communauté d'origine. ${ }^{7}$ Il est intéressant de noter que ce code de conduite est né en pleine controverse : un certain nombre de polémiques occupaient alors la scène politique régionale. Bethwel Kiplagat, jugé responsable du Wagalla massacre, ${ }^{8}$ est destitué par la Truth Justice and Reconciliation Commission (TJRC) qu'il préside en novembre 2010. Le Kalenjin Council of Elders, ${ }^{9}$ autorité dont dépend le Nandi Kaburwo Council of Elders, prend alors sa défense en la personne de Josuah Sang, nominé à la tête du Kalenjin Council of Elders peu après l'adoption du NCPCC. Kethwell Kiplagat est finalement renommé à la tête de la TJRC en avril 2012 grâce à l'influence du Ministre de la Justice Eugene Wamalwa. Au même moment, environ deux semaines avant l'entrée en fonction de Josuah Sang, le Kalenjin Council of Elders refuse de reconnaître William Ruto comme le candidat favori des Kalenjin à la présidentielle, alors jugé par la Cour Pénale

5 C'est Ben, un jeune chanteur nandi qui m'a transmis ce document. Lors de la campagne politique, il a été rémunéré par des politiciens locaux pour écrire une chanson en kalenjin intitulée " Uhuruto », dont le clip a été tourné à Kapsimoto gardens dans les jardins luxuriants de l'une des nombreuses propriétés du Président Moi dans la région. Dans cette chanson de campagne, Ben chante les louanges des leaders de demain et de leur alliance politique, Uhuru Kenyatta et plus particulièrement de William Samoei Ruto, jeune leader de l'United Republican Party (URP), parti dont l'électorat de base est principalement kalenjin et qui a beaucoup de succès dans la région. C'est lors de la campagne, en tant que partisan, qu'il a reçu l'un des exemplaires du NCPCC. 6 Les bases de ce code de conduite ont été arrêtées lors d'une réunion des différentes community based organizations du comté, sous la houlette de Denis Kogo, alors président du Rift Valley Development Trust. Pour plus de détails sur la création de ce code de conduite, voir l'article « NGO Sending Shivers on Political Leaders in Nandi ». Lien: http://lawrencekoech.wordpress.com/2011/10/10/ngo-sending-shivers-on-politicalleaders-in nandi/

7 "Vision and Mission: create a safer future for the world by working with local communities to identify their societal problems. » Source : site officiel de la CCDD. Lien : http://www.ccddkenya.org/

8 Kethwel Kiplagat est une personnalité kalenjin proche du Président Moi : il a occupé différents postes administratifs et diplomatiques d'importance de 1978 à 1983 et a également acquis une aura internationale en tant que chairman du Africa Peer Review Mechanism of the New Partnership for Africa's Development (NEPAD) et membre d'International Crisis Group (ICG). Nommé Chairman de la Kenya's Truth Justice and Reconciliation Committee en août 2009, il est forcé de démissionner en novembre 2010 en raison d'un rapport qui fait état de son rôle trouble dans l'assassinat de l'ancien Ministre des Affaires étrangères Robert Ouko ainsi que dans le massacre d'environ 3000 kényans musulmans d'origine somalienne perpétré par les forces de sécurité kényanes à Wagalla, North Eastern Province, en 1984.

9 Le Kalenjin Council est basé à Eldoret. Au regard de la loi kényane, qui reconnaît le droit aux communautés infra-nationales d'être représentées par un conseil des anciens disposant pour ce faire de la possibilité de se constituer en personne morale, le Nandi Kaburwo Council of Elders est l'un des dix bororosiek ou sousgroupes du Kalenjin Council of Elders. 
Internationale (CPI) pour crime contre l'humanité. ${ }^{10}$ Cette double prise de position est vivement critiquée, perçue par certains comme la consolidation des réseaux clientélistes kalenjin, Bethwel Kiplagat étant un ancien de l'administration Moi, et analysée par d'autres comme une trahison, la candidature de William Ruto ainsi que son parti l'United Republican Party (URP) symbolisant l'unité kalenjin. Face à la polémique, Josuah Sang déclare que le conseil des anciens est une institution neutre qui n'a pas à se prononcer en faveur d'un parti plus que d'un autre et s'engage à travailler main dans la main avec la NCIC pour maintenir la paix dans la région pendant la campagne électorale. ${ }^{11}$

Ainsi, le Nandi Kaburwo Council of Elders se présente comme une institution neutre, dont la légitimité est suffisante pour participer au vaste effort national pour la tenue d'élections pacifiques et l'avènement d'un ordre politique nouveau. Le code de conduite proposé est alors présenté comme « a set of rules of behavior for political parties and their supporters relating to their participation in an election process, to which the parties ideally will voluntarily agree, and which may subsequent to the agreement be incorporated in law ». Les politiciens et leur équipe de campagne doivent signer ce code en tant que garants des droits et libertés des citoyens énoncés par la constitution (I. Art $1 ; 3) .{ }^{12}$ Un principe central hérité de la séparation des pouvoirs y est rappelé : les candidats doivent mobiliser leurs propres ressources pour faire campagne et ne peuvent faire appel aux pouvoirs de l'État (I. Art 7). Il s'agit ici de définir ce qu'est d'être un «bon leader » ainsi qu'une nouvelle forme de leadership, autour de l'idée que des élections bien conduites seront garantes d'une plus grande démocratisation du pays. La définition du rôle attribué aux représentants élus illustre cette volonté de rupture : le « bon leader » est avant tout défini par la négative. En effet, le code consiste principalement en une succession d'interdictions et la prohibition d'un certain nombre de comportements portant atteinte à la cohésion nationale : interdiction des appels à la violence ou hate speech (I. Art 10 ; 18), de la corruption, de l'achat de voix par la distribution d'argent ou par des fausses promesses faites aux électeurs (I. Art 20), de la consommation d'alcool, des techniques

10 Pour plus de détails, consulter l'article du journal The Star, «Kalenjin Council of Elders replaces Seii as chairman », 28/04/2012: "Sang takes over just two weeks after the council made a controversial decision to back Eldoret North MP William Ruto as Presidential candidate during the recent Kamatusa meeting in Eldoret. [...] Sang said the council had also resolved to restore confidence in the Truth Justice and Reconciliation Commission following the reinstatement of Ambassador Bethwel Kiplagat as chairman of the truth body. " Lien : http://www.the-star.co.ke/news/article-20536/kalenjin-council-elders-replaces-seii-chairman

11 Ibid. "[...] Yesterday Sang said they will remain politically neutral and work with all political parties especially their presidential candidates. 'We will recognise and respect all political parties in particular their flag-bearers since the elders council is non-partisan', said Sang soon after he was elected to head the council which is a key community organ in the Kalenjin community. [...] Sang said they had also resolved to work with the NCIC and the provincial administration to ensure that all those seeking elective positions avoid using provocative language during the election campaigns. "

12 Afin de simplifier les références faites aux articles du code de conduite, celui-ci sera divisé en trois parties : je me référerai au Preamble et au Background comme à l'introduction du code. I. Art 1 correspond au premier article de la première partie Regulations and Commitments, et II. Art 1 au premier article de la deuxième partie Compliance and Enforcement. 
d'intimidation (I. Art 27), des violences entre camps partisans (I. Art $11 ; 13 ; 17 ; 20$ ). Enfin, il est clairement rappelé que le jour de l'élection, les candidats ne sont pas autorisés à faire campagne (I. Art 23 ; 25). Bien que la plupart des élus avaient déserté la ville le dernier jour de la campagne, laissant planer un climat d'apparence calme mais très pesant, certains candidats faisaient toujours campagne, distribuant des enveloppes pleines de billets dans les parties les plus reculées du comté, comme ce fut le cas à Kapkangani. ${ }^{13}$

Au-delà de la redéfinition de ce que doit être un «bon leader » en temps électoral, ce code a pour vocation de consolider la relation qui unit les citoyens à leurs représentants en mettant en place des principes éthiques que les futurs élus locaux et régionaux s'engagent à respecter dès le début de leur campagne et non simplement au début de leur mandat. Cette relation de confiance permettrait de créer un sens du devoir et de l'éthique chez les candidats, ce qui augmenterait par conséquent leur légitimité à gouverner aux yeux de l'ensemble des citoyens kényans, leurs opposants y compris. Cette acceptation des résultats est pensée comme la condition sine qua non pour que le scénario de 2007 et les affrontements qui ont accompagné les accusations de fraude électorale ne se reproduisent pas. Par ailleurs, ce code fonctionne comme une sorte de " bible politique " promue par les anciens et à laquelle les politiciens ont juré allégeance. Ils deviennent ainsi les " prédicateurs de la bonne parole $",{ }^{14}$ en s'engageant à diffuser le plus largement possible les préceptes évoqués par le code (II. Art 1) qui doit être présenté comme un élément central de leur campagne (II. Art 2). Le non-respect de ce code est encadré par les autorités kényanes au premier rang desquelles se trouve l'Independent Electoral and Boundaries Commision (IEBC). Toute transgression est passible d'emprisonnement (II. Art. 3) et une série de sanctions graduelles applicables sous l'autorité du responsable régional ou local des élections (Electoral Chief) sont prévues pour le candidat et son parti. Le nonrespect de ce code expose également les candidats et leurs partisans aux représailles du Nandi Kaburwo Council of Elders, dont la fonction est également de punir, par le biais d'ensorcellements, ceux qui vont à l'encontre du bien commun de la communauté nandi. ${ }^{15}$

13 Je parcourais le comté la veille des élections et ai pu observer à cette occasion la visite d'un candidat à Kapkangani, petite localité située à la frontière nord du comté Nandi, sur la route de Kakamega.

14 La campagne électorale a été marquée par de nombreux discours politiques de type téléologiques. Voir à ce sujet les travaux d'Yvan Droz et d'Hervé Maupeu sur la pentecôtalisation de la politique au Kenya.

15 Les elders ont également pour fonction de jeter des mauvais sorts sur certaines mauvaises personnes désignées par la communauté. Ce fut le cas l'an passé lorsqu'un couple gérant une plantation de thé fut tué par des voleurs. Les elders réalisèrent alors une cérémonie sur le lieu du crime, jetant un sort mortel aux criminels en présence d'une foule importante. Kenya citizen TV, « Nandi Elders perform ceremony to curse family's killers ». Lien vers la vidéo de la cérémonie : http://youtu.be/ET-5SjiDXmU 
En ce sens, ce code de conduite, s'il rappelle le Electoral Board Code of Conduct diffusé par la commission électorale kényane (IEBC) ou les " goodwill ambassadors $»^{16}$ désignés pour porter des messages de paix et d'unité par la National Cohesion and Integration Commission (NCIC), est une initiative originale. En effet, il est rare que des councils of elders aient mis en place ce type de « directives » dans d'autres comtés du Kenya. Comment se fait-il que le Nandi Kaburwo Council of Elders ait souhaité la rédaction d'un code de conduite en temps électoral, qui à terme devrait fonctionner comme un véritable « contrat social» entre les électeurs et leurs élus ?

Dès l'introduction, il est précisé que ce code est écrit au nom du peuple nandi, présenté comme une entité rationnelle, consciente des opportunités fournies par le nouveau système administratif décentralisé et de l'impact global des enjeux politiques passés et présents sur le développement du comté. Le développement économique de la communauté, qui a largement été ralenti par les violences post-électorales de 2007-2008 ayant particulièrement touché la région, est un argument redondant du code de conduite. L'industrie du thé y a été ralentie, les ouvriers nandi mais aussi d'origines luo ou kisii ayant dû fuir, tout comme un certain nombre d'investisseurs. Les membres du council of elders ont un certain statut social et économique au sein de la communauté nandi : nombre d'entre eux font partie des entrepreneurs et des investisseurs de la région et occupent des postes clés au sein de l'administration, des médias ou du secteur agro-alimentaire. En ce sens, le Nandi Kaburwo Council of Elders fonctionne comme un club de notables, ${ }^{17}$ un groupe d'intérêt fermé dont les portes ne s'ouvrent que par une procédure de cooptation discutée en amont par les pairs. Cette idée se confirme lorsqu'on s'intéresse plus particulièrement à certains préceptes du code de conduite, qui soulignent des intérêts purement économiques : l'introduction mentionne explicitement que ce code s'inspire de la déontologie de secteurs professionnels particuliers (healthcare, finance and legal services), les contribuables y sont présentés comme une faction distincte du corps électoral (Voters and taxpayers) et la mission d'un " bon leader » est également de susciter stabilité et confiance dans la qualité des services et le dynamisme du comté («instills confidence in the Nandi County's professional standards and performance »).

16 Ces 25 ambassadeurs de la paix, d'origines ethniques et professionnelles variées (militaires, athlètes, businessmen, intellectuels et artistes), ont été nommés par la NCIC en juillet 2012 dans le cadre de la campagne Kenya Kwanza ( «enya comes first »). Bien que leur rôle soit avant tout symbolique, ils avaient pour mission de diffuser des discours d'unité et de paix pour que les élections de mars 2013 se déroulent sans heurts.

17 Sur le fonctionnement des groupes de notables locaux et plus particulièrement à Nandi Hills, voir la thèse récemment soutenue par Dominique Connan, La Décolonisation des clubs kényans. Sociabilité exclusive et constitution morale des élites africaines dans le Kenya contemporain, 27 janvier 2014. 
Ainsi, le Nandi Kaburwo Council of Elders s'exprime moins au nom d'une communauté nandi supposée homogène ${ }^{18}$ qu'en tant que groupe de notables locaux. Cette idée est renforcée par les modalités selon lesquelles un membre de la communauté acquiert le statut d'elder. Les entretiens que j'ai pu mener auprès de plusieurs elders soulignent que ces derniers s'accordent sur trois points ${ }^{19}$ : le candidat doit être âgé de plus de 55 ans, avoir joué un rôle structurant dans la communauté et être intronisé selon une procédure ritualisée. ${ }^{20} \mathrm{E}$ réalité, les règles pour accéder au statut d'elder apparaissent plus flexibles. Le conservateur du musée de Koitalel Samoei par exemple, âgé d'une trentaine d'année, a rejoint le Nandi Kaburwo Council of Elders en 2007 au moment où il a pris ses fonctions au sein du musée. De même, l'un des descendants de Koitalel, journaliste pour le magasine Kass et auteur de la première biographie (très largement romancée) de son aïeul, a également été intronisé à l'unanimité sans respecter la limite d'âge imposée.

Pourtant, ce code a un sens particulier pour de nombreux membres de la communauté nandi car il fait écho à une croyance collective, présente dans les conversations quotidiennes et les brochures, les rituels et les discours des membres du Nandi Kaburwo Council of Elders. Cette croyance se base sur une mémoire collective mise en récit dans le musée de Koitalel Samoei : un imaginaire guerrier qui serait la pierre de touche d'un âge d'or nandi. ${ }^{21}$ Cet âge d'or aurait pris fin avec un sort lancé il y a plus d'un siècle par leur Oorgoiyot, c'est à dire un chef à la fois politique, militaire et spirituel qui régnait sur les différents sous-groupes nandi, ayant eux-mêmes leurs propres chefs. La communauté aurait été maudite à la suite de la perpétration du meurtre de leur leader de l'époque, Kimnyolei, dont la communauté s'était détournée. Les chefs militaires sous la protection de Kimnyolei avaient refusé d'écouter les oracles de ce dernier, qui empêchaient les différents clans de la communauté de chasser ou de capturer du bétail. Plusieurs chefs désobéirent. En chemin, les guerrier nandi trouvèrent une carcasse de buffle et décidèrent de manger la bête. Tous moururent peu après. Ils décidèrent alors de tuer Kimnyolei, persuadés qu'il avait prononcé un sort mortel à leur encontre, refusant de croire que c'était les Dieux eux-même qui les avaient punis. Omniscient et pouvant prédire l'avenir, Kimnyolei anticipa leur trahison et avant de succomber à leur attaque, il

18 Les Nandi étant bien souvent perçus à l'échelon national comme une communauté essentiellement composée d'employés agricoles peu éduqués de l'industrie du thé, je nuance ici le propos en me référant à la littérature sur l'identité collective et son durcissement lorsqu'elle est envisagée à des fins politiques, et plus particulièrement les travaux de M.-C. Lavabre et de D.-C. Martin.

19 Ces trois points sont formalisés par la constitution du Kalenjin Council of Elders, qui définit le statut de l'association ainsi que son fonctionnement. Voir plus particulièrement l'article 6 de la constitution : Membership of the Council, life-membership, co-optation and entrance fees.

20 Il s'agit d'une cérémonie où l'on offre au nouveau membre un «bâton de pouvoir » (signe distinctif d'une personnalité remarquable) taillé dans de l'olivier sauvage et au cours de laquelle ses mains et ses pieds sont oints de graisse mélangée à du lait caillé.

21 Les Nandi sont régulièrement présentés dans les brochures du Nandi Kaburwo Council of Elders comme un peuple ayant dominé la région grâce à leur supériorité militaire, leur structure sociale très hiérarchisée et leur unité garantie par l'Orkoiyot, figure type du « bon leader » dévoué à son peuple, omniscient et choisi par les dieux. 
jeta une malédiction sur la communauté tout entière qui ne devrait plus jamais connaitre l'harmonie : "He had accursed the Nandi that their leadership will break like a pot $» .^{22}$ Ce mauvais sort continuerait de hanter tout particulièrement les Nandi et les Kipsigis supposés être les descendants des clans des chefs militaires qui désobéirent à Kimnyolei et planifièrent son assassinat, qui n'ont pas connus de " bon leader» depuis Koitalel Samoei, leur dernier Oorgoiyot qui mena une guerre contre les britanniques pour la possession de l'escarpement Nandi au début du XXème siècle. Cette croyance collective est qualifiée par Gabrielle Lynch de "narratives of communal grievance » qui stipulent que les élites élues s'acharnent à marginaliser la communauté nandi dans la sphère politique. ${ }^{23}$ L'existence de ce récit s'explique par le rapide turnover des députés issus des communautés Nandi et Kipsigis dans les années 1980, encouragé par le président Moi afin d'éviter qu'une opposition organisée puisse se mettre en place dans la région. ${ }^{24}$ Ce récit s'explique également par la forte croyance il existait qu'auparavant un lien de confiance transcendantal entre l'Oorgoiyot et sa communauté, qui garantissait permettait aux Nandi le succès de toute entreprise commune et une supériorité sur les autres groupes ou sous-groupes de la région, puissance et harmonie qui auraient été perdues lorsque la communauté se retourna contre son leader.

Ainsi, qu'il s'agisse d'une trahison du leader par la communauté ou de la communauté par le leader, la défiance qui lie électeurs et élus constitue le terreau sur lequel des initiatives comme le Nandi Political Code of Conduct voient le jour. Ce code n'est pourtant que partiellement respecté : au-delà des écarts constatés en temps électoral, presqu'un an jour pour jour après l'élection du gouverneur du comté Nandi Cleophas Lagat (URP), une manifestation accusant ce dernier de corruption s'est tenue à Kapsabet le 25 mars 2014. ${ }^{25} \mathrm{Ce}$ code de conduite, loin de se résumer à une simple entreprise d'acteurs nationaux (IEBC), internationaux (l'ONG CCDD) ou locaux (Nandi Kaburwo Council of Elders), constitue un formidable outil de compréhension des aspirations politiques d'une communauté dont la marginalité et l'unité sont modelées par des récits et croyances issues d'une mémoire collective en perpétuelle reconstruction. Au vu des enjeux politiques que ce code de conduite représente pour la communauté nandi et de l'importance qu'il aura dans mon travail de thèse, il m'a semblé important de le publier ici en ce qu'il illustre l'articulation complexe des politiques de réconciliation nationale aux enjeux précis portés par les élections locales et régionales pour une communauté ayant sa propre conception en termes de leadership. Plus généralement, ce document original permet de s'interroger sur le rôle joué au Kenya par les councils of elders au niveau local, tant d'un point de vue

22 Cette histoire est explicitée par l'une des brochures du Nandi Kaburwo Council of Elders, The Nandi-Talai propiation convention held on $31^{\text {th }}$ December under the auspices of the Nandi Kaburwo Council of Elders. Background on the information of the Talai Clan and the road to propiation, healing and reconciliation.

23 Gabrielle Lynch, I say to you. Ethnic Politics and the Kalenjin in Kenya, 2011, p. 128.

24 Gabrielle Lynch, Ibid, p. 129.

25 Cet événement est relaté par plusieurs blogs nandi (comme le groupe The Nandi County News sur facebook) ou encore l'article du journal The Star, 25/03/2014, « Nandi police stops demo by civil groups ». Lien : http:// www.the-star.co.ke/news/article-160164/nandi-police-stop-demo-civil-groups 
social et identitaire qu'économique et politique.
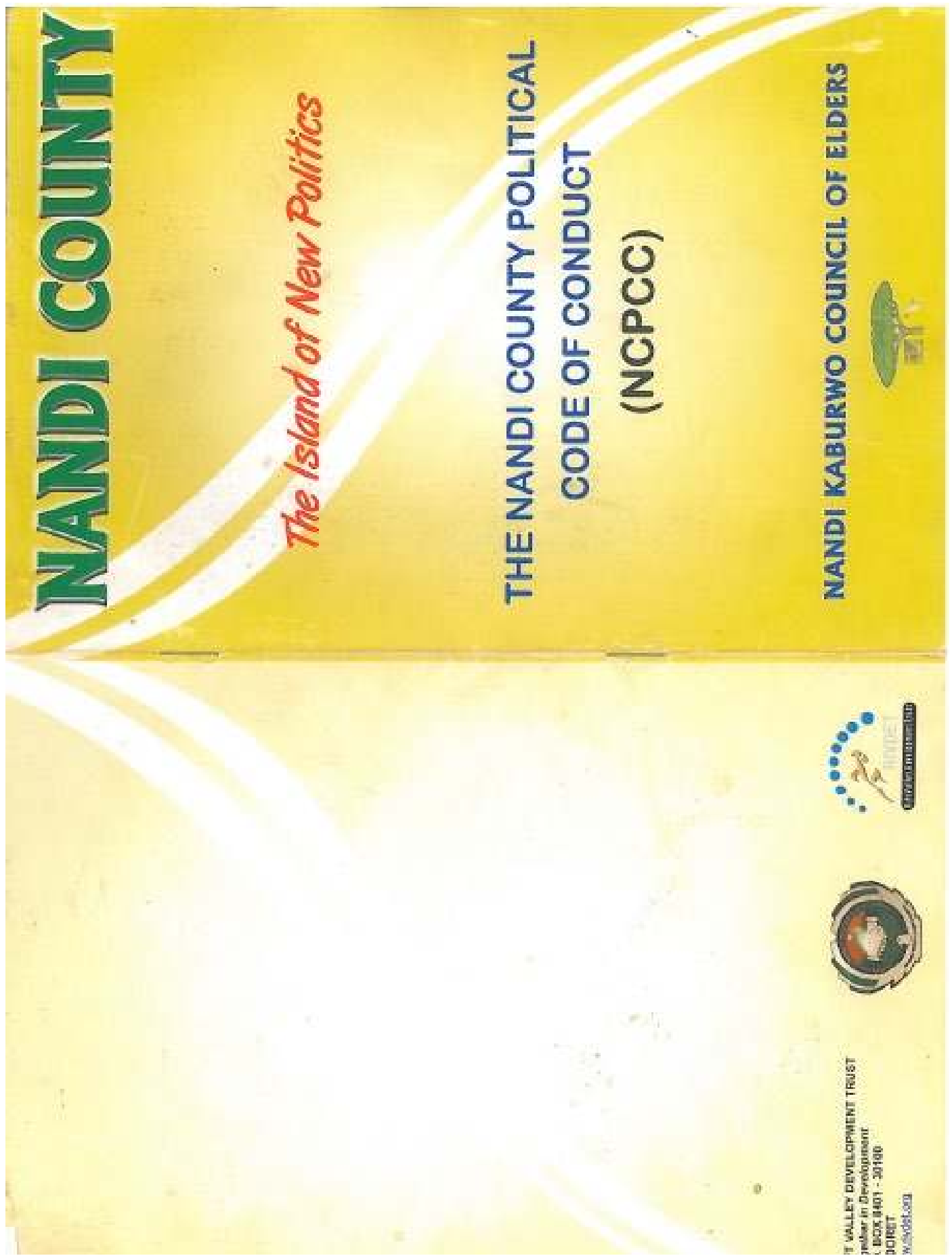
Les Cahiers d'Afrique de l'Est

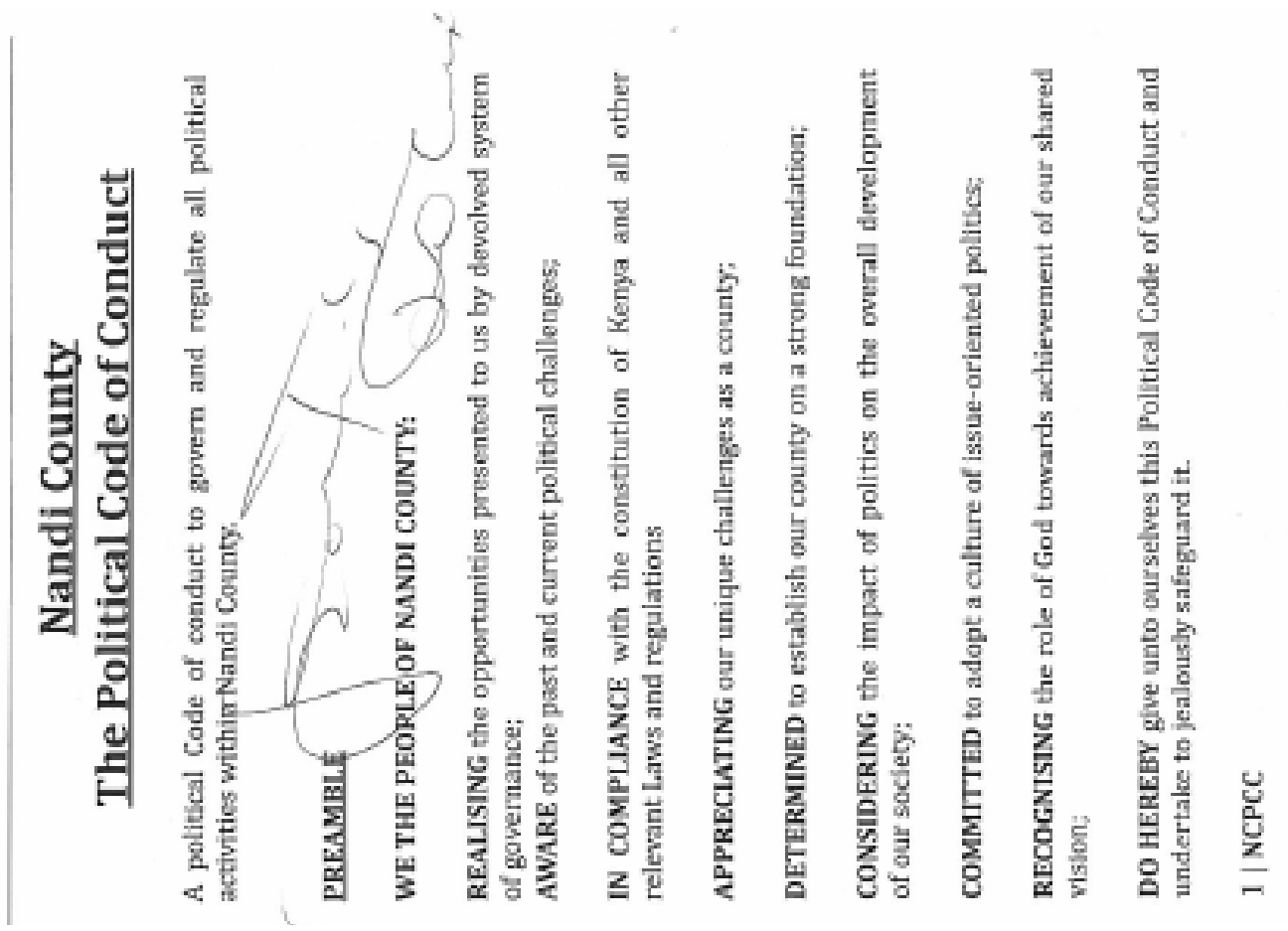




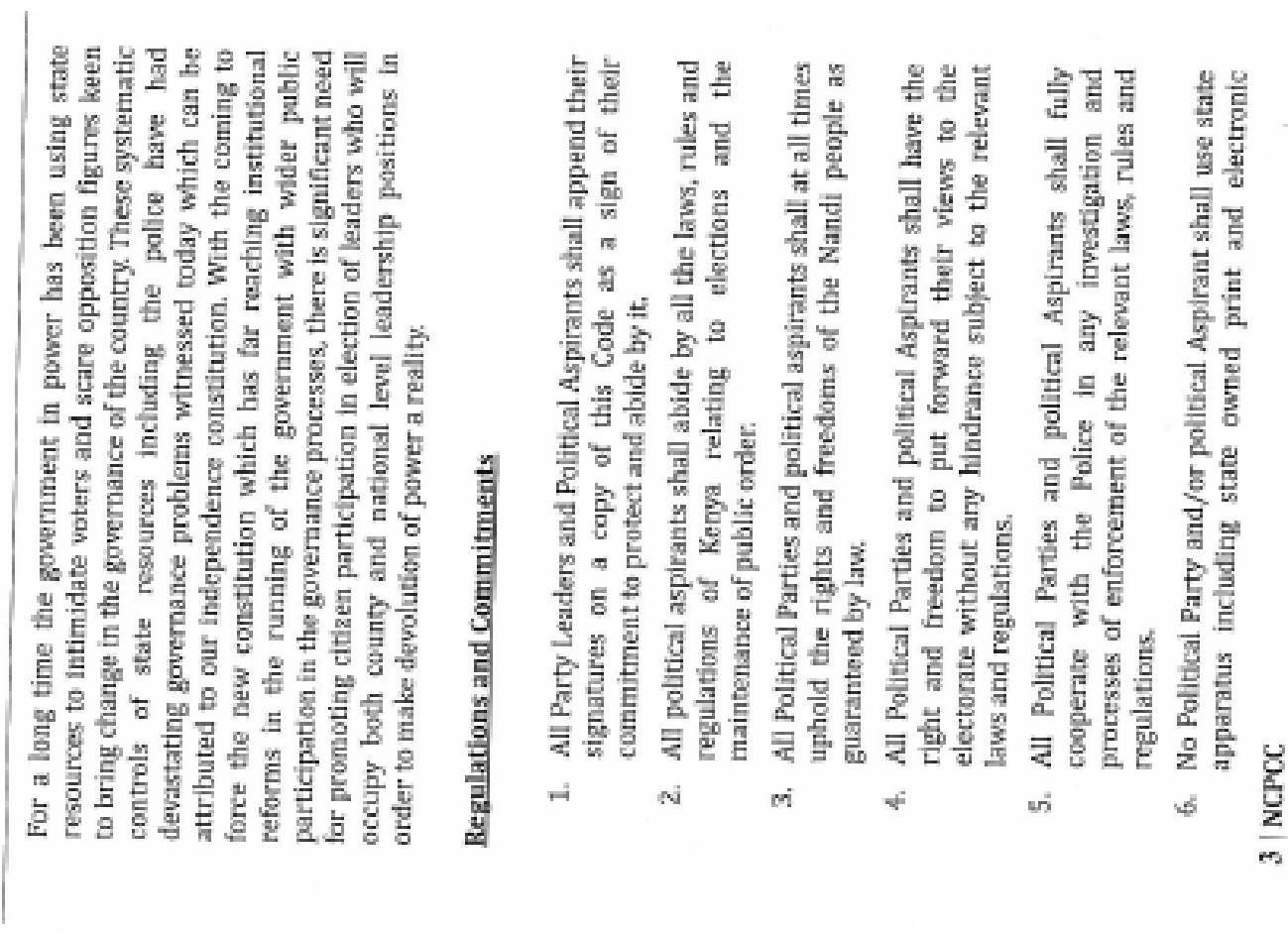

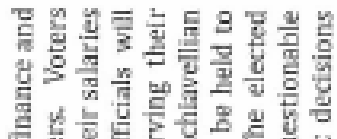

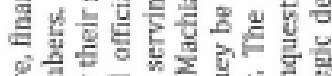

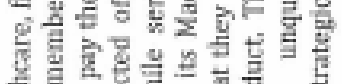

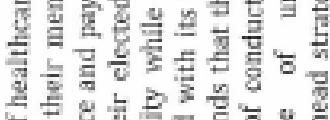

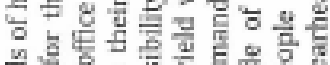

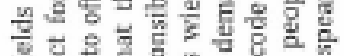

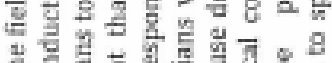

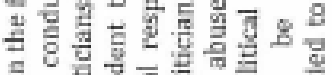

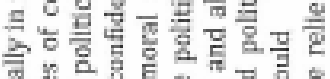

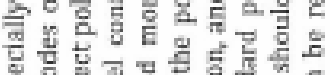

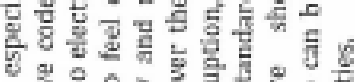

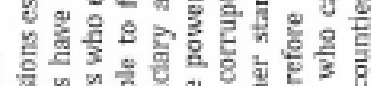

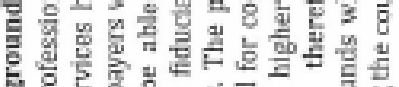

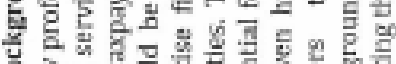

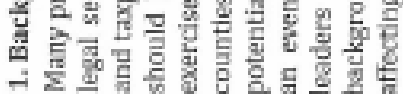

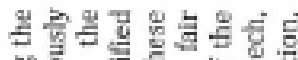

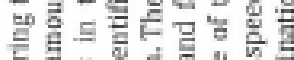

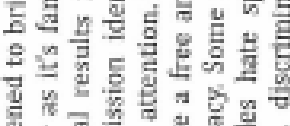

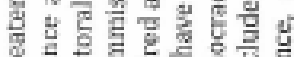

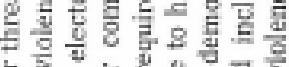

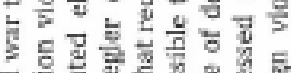

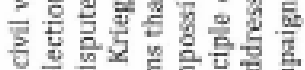

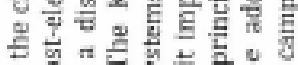

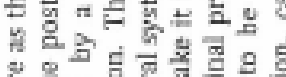

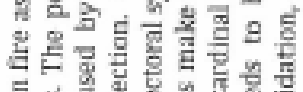

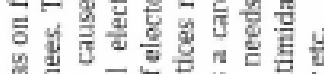

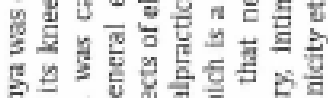

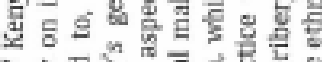

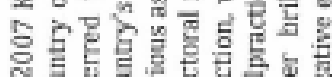

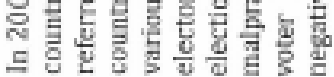

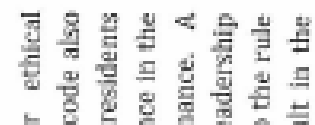

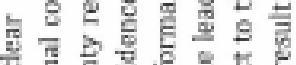

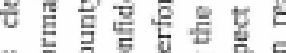

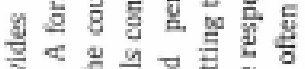

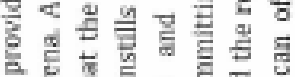

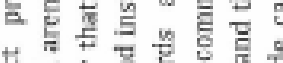

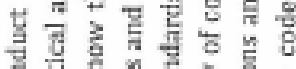

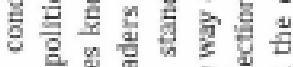

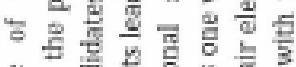
늠. 몰 ن

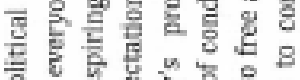

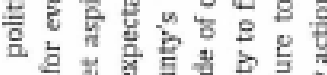

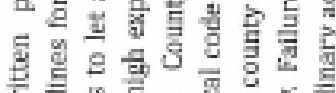
는 든

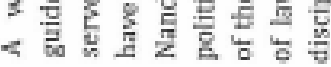




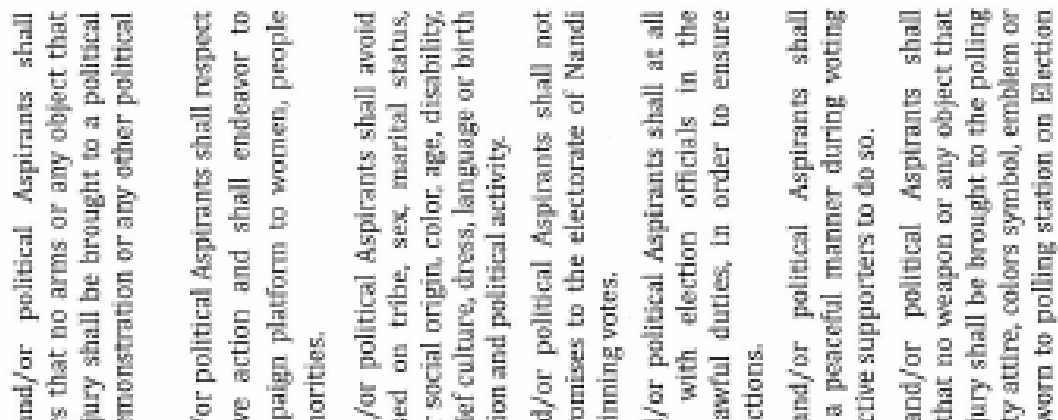

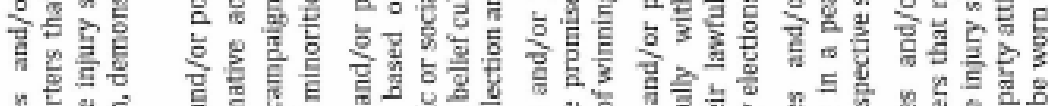

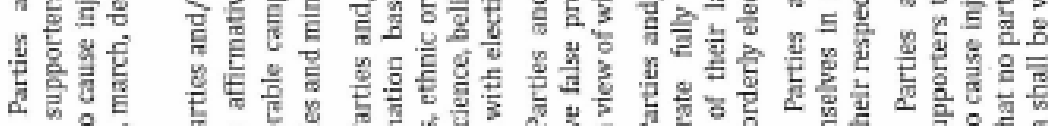

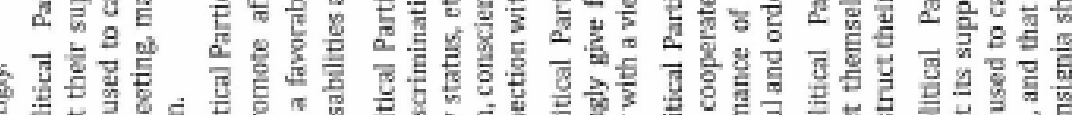

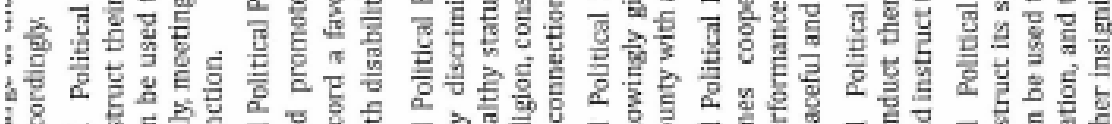
| \&

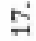
$\stackrel{\leftrightarrow}{3}$ 㞼

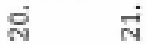
तู่ लู่

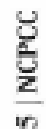

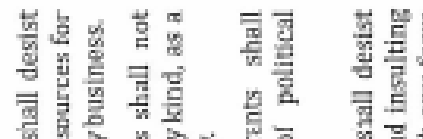

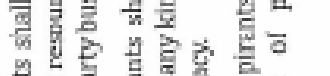

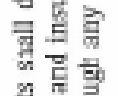

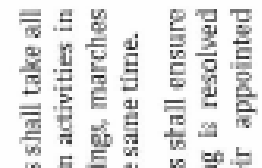

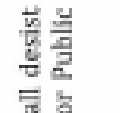

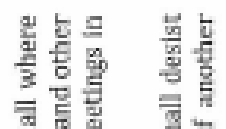

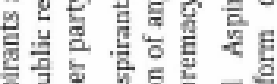

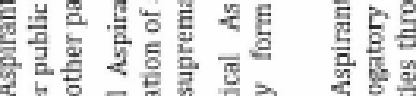

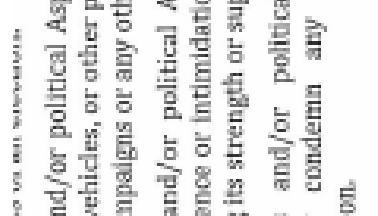

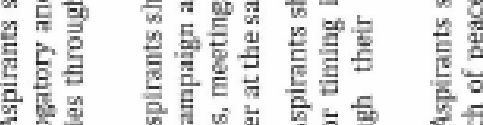

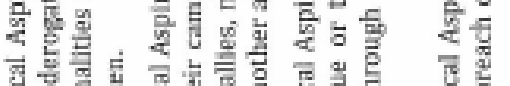

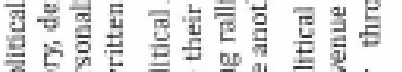

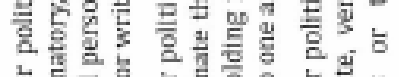
$\frac{\mathrm{E}}{\mathrm{E}} \frac{\mathrm{d}}{\mathrm{g}}$

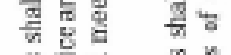

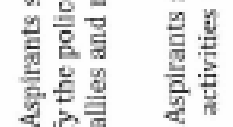
절 볼

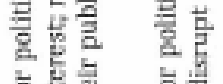

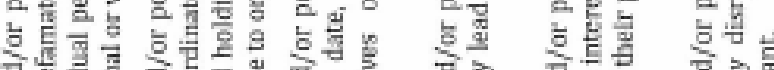

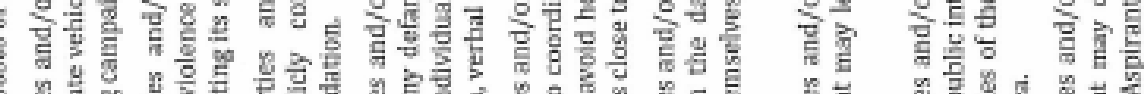

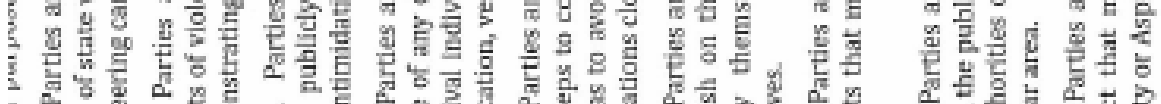
1

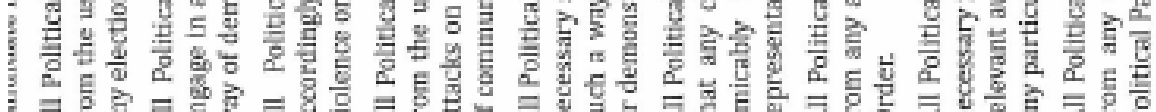

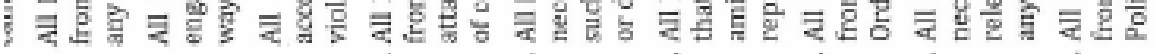
i $\infty$ oi 实 일 ๓่ 器 


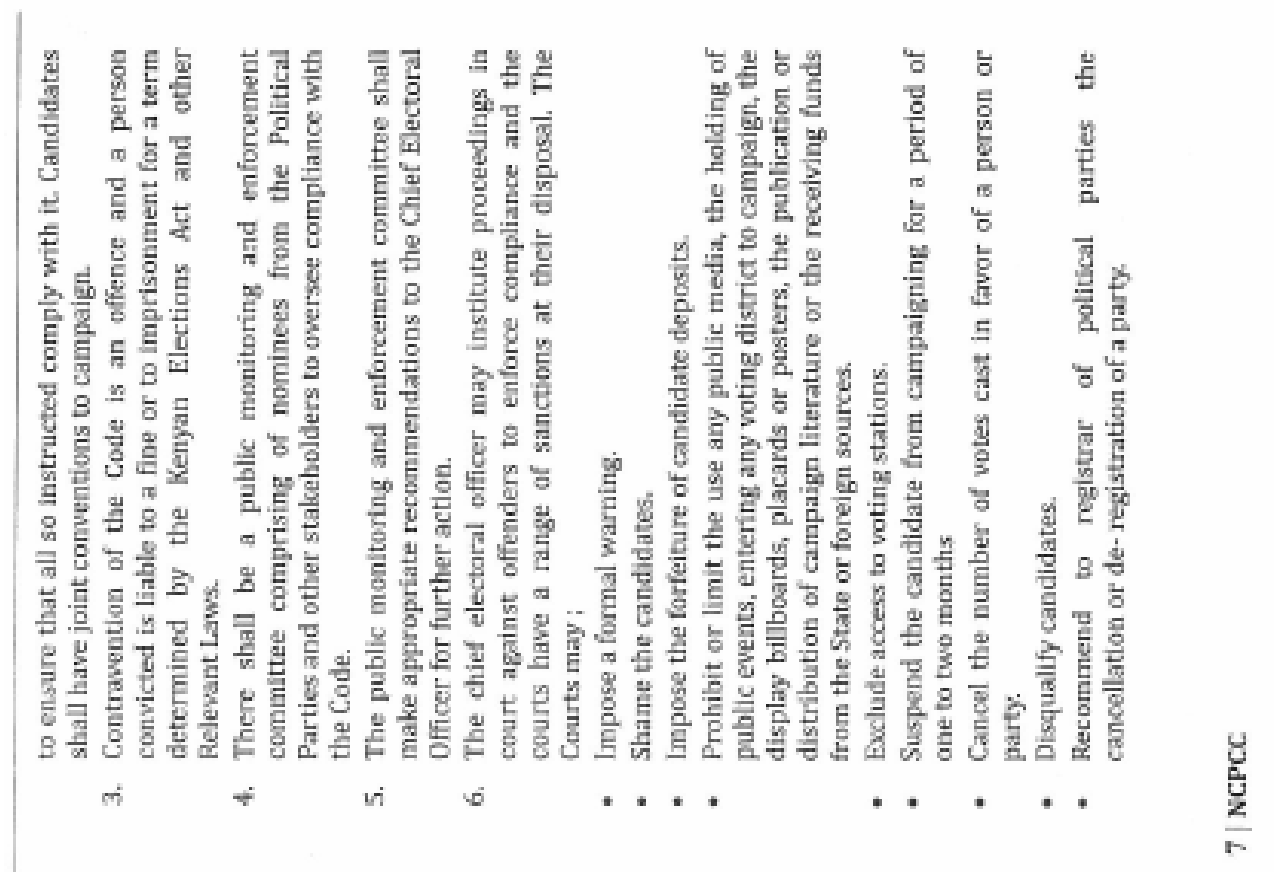

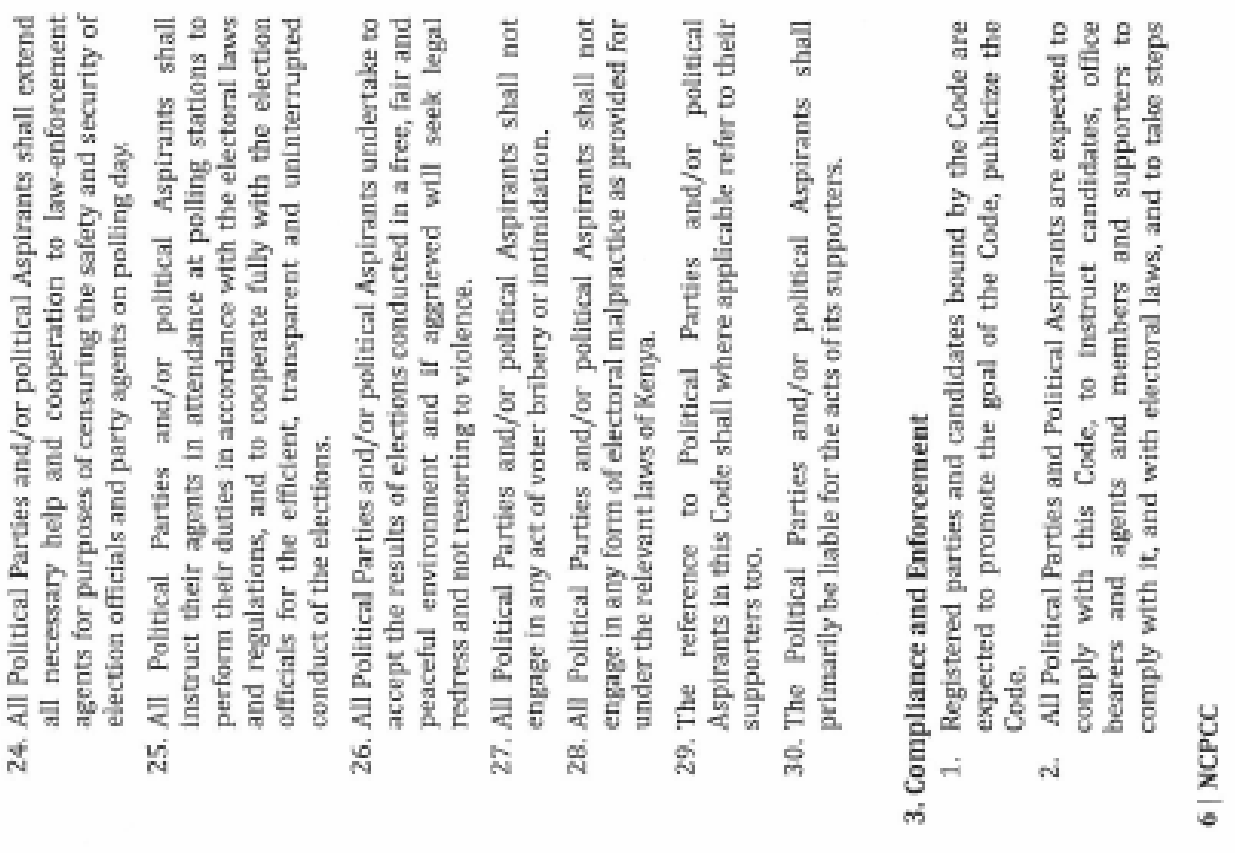




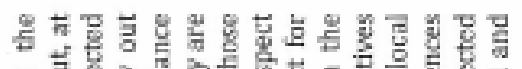

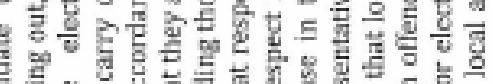

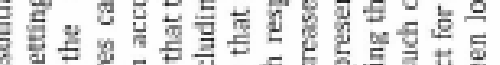

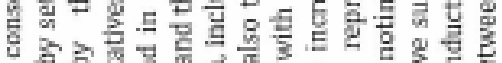

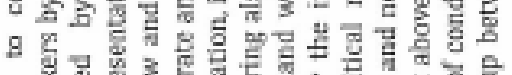

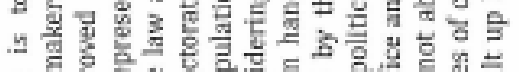

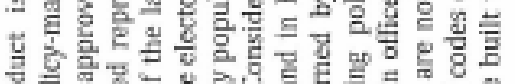

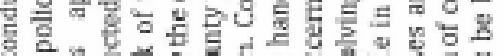

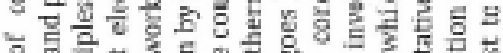

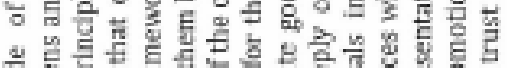

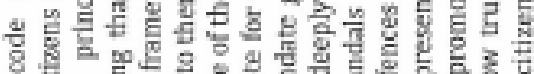

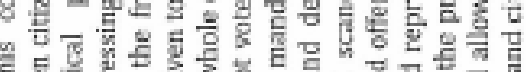

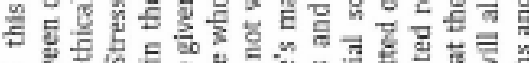
을
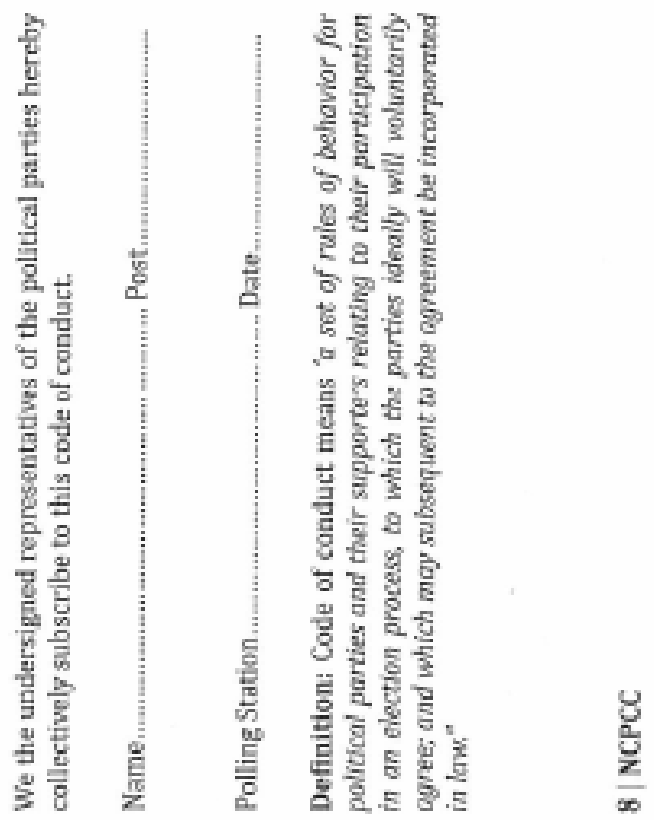